\title{
Reduced-Order Modeling of Turbulent Reacting Flows with Application to Ramjets and Scramjets
}

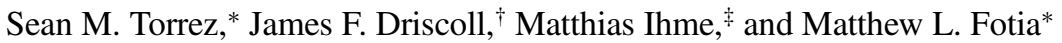 \\ University of Michigan, Ann Arbor, Michigan 48109
}

DOI: $\underline{10.2514 / 1.50272}$

\begin{abstract}
A new engine model has been developed for applications requiring run times shorter than a few seconds, such as design optimization or control evaluation. A reduced-order model for mixing and combustion has been developed that is based on nondimensional scaling of turbulent jets in crossflow and tabulated presumed probability distribution function flamelet chemistry. The three-dimensional information from these models is then integrated across cross-sectional planes so that a one-dimensional profile of the reaction rate of each species can be established. Finally, the one-dimensional conservation equations are integrated along the downstream axial direction and the longitudinal evolution of the flow can be computed. The reduced-order model accurately simulates real-gas effects such as dissociation, recombination, and finite rate chemistry for geometries for which the main flow is nearly onedimensional. Thus, this approach may be applied to any flowpath in which this is the case; ramjets, scramjets, and rockets are good candidates. Comparisons to computational fluid dynamics solutions and experimental data were conducted to determine the validity of this approach.
\end{abstract}

\section{Introduction}

$\mathbf{T}$ HIS work addresses the need for an improved control-oriented model of a dual-mode ramjet/scramjet propulsion system. Improvements are needed to include more realistic estimates of the losses of the propulsion efficiency due to shock wave interactions in the inlet, as well as due to gas dissociation and incomplete combustion in the combustor section. One problem is that previous lowerorder propulsion models [1-3] do not include the losses due to multiple shock interactions, gas dissociation, and incomplete combustion caused by finite rate chemistry. This is a serious problem, because the main advantage of a scramjet engine over a ramjet is that the scramjet reduces losses due to internal shock waves and gas dissociation [4]. That is, the scramjet eliminates the need for strong internal shock waves to decelerate the gas to subsonic conditions and maintains lower static temperatures than a ramjet, which reduces the dissociation losses. The present effort addresses previous shortcomings by including both of these types of losses into a code called MASIV. MASIV consists of several reduced-order models (ROMs). One is an inlet ROM that computes losses due to multiple shock/ expansion wave interactions; this ROM is described elsewhere [5]. The other is a fuel/air mixing/combustion ROM that is the focus of the present paper. MASIV has been incorporated into a larger hypersonic vehicle (HSV) code, which is available without charge and without International Traffic in Arms Regulations restrictions.

Since computational fluid dynamics (CFD) codes take many hours to reach solutions for reacting flows, they are difficult to apply to problems in which a large number of solutions are required. A tool that can solve these configurations in a short time to acceptable accuracy is highly desirable for control and design applications, such as control evaluation, and multidisciplinary optimization (MDO). The proposed one-dimensional method [6-10] solves for the heat release distribution for both subsonic and supersonic internal flows.

Received 23 April 2010; revision received 31 August 2010; accepted for publication 31 August 2010 . Copyright $\odot 2010$ by Sean M. Torrez. Published by the American Institute of Aeronautics and Astronautics, Inc., with permission. Copies of this paper may be made for personal or internal use, on condition that the copier pay the $\$ 10.00$ per-copy fee to the Copyright Clearance Center, Inc., 222 Rosewood Drive, Danvers, MA 01923; include the code $0748-4658 / 11$ and $\$ 10.00$ in correspondence with the CCC.

${ }^{*}$ Research Assistant, Department of Aerospace Engineering. Student Member AIAA.

${ }^{\dagger}$ Professor of Aerospace Engineering, Department of Aerospace Engineering. Fellow AIAA.

ॠAssistant Professor of Aerospace Engineering, Department of Aerospace Engineering. Member AIAA.
Most control evaluation codes and some MDO routines use ROMs in some capacity in order to provide solutions in a reasonable amount of time. ROMs are typically based on approaches employing dimensional reductions in which detailed or high-fidelity simulations are approximated through a set of basis functions or lookup tables [11]. ROMs usually do not compete with CFD, elementary combustion, finite element, or other high-fidelity simulations, but instead use these high-fidelity solutions at run time. While these types of ROMs provide a rapid way to generate solutions, they are confined to the fixed geometry and the limited range of validity for which the tabulated results were generated. The accuracy and range of validity of each ROM must be carefully quantified by making comparisons to high-fidelity CFD or experimental findings.

The present work treats the problem differently from the proper orthogonal decomposition (POD) approach; it reduces the dimensionality of the physics retained in the problem so that the entire code can be run in a short time. This makes it possible to solve the entire flowfield, rather than fully relying on pretabulated solutions, and it means that run conditions are not constrained to be between the bounds of pretabulated cases for the physics of the simulation to be accurate, since the conservation laws are solved directly. They are not reduced into an interpolation or a regression scheme.

The combustor code solves a set of differential and algebraic equations in space [12], marching axially through the combustion duct. Since combustion in most engines is mixing-limited rather than reaction-rate-limited, 3-D jet mixing must be computed. However, since we only allow differential equations containing derivatives with respect to the axial distance coordinate, we use an algebraic jet spreading scaling relation [13]. This amounts to using a ROM for jet spreading characteristics, but the solution is based on flowfield similarity rather than a lookup table, so it is valid for a wide range of flow conditions that have jet-wake-stabilized flames.

The combustion model considers finite rate chemistry within the steady laminar flamelet model (SLFM) [14], which considers each point in the flame and maps to it the solution of a corresponding counterflow flame. To account for the turbulence/chemistry interaction, this SLFM formulation employs a presumed probability distribution function (PDF) closure model. The SLFM includes the effects of strain rate, species diffusion, and momentum diffusion with changing duct velocity and fuel jet velocity. The PDF closure accounts for the effect of turbulence. This allows us to generate a three-dimensional reaction-rate field, which we then spatially average over transverse planes to create a one-dimensional reactionrate profile.

In this work, we compare results of the MASIV ROM to CFD solutions from the commercially available FLUENT [15] code and to 
profiles of wall pressure and heat release rates measured by Micka et al. [16]. The results shown are from the current version of MASIV, which simulates either scram mode combustion or ram mode and uses an ad hoc method to jump over the singularity at Mach 1. The ram-scram transition was not considered, although the equations that govern this transition have been previously described by the authors [7] and will be implemented in future versions of the code.

\section{Model Description}

The model is designed to simulate flowpaths that have a general 1-D character but contain regions where 3-D mixing and combustion occur. For example, in a dual-mode ramjet/scramjet, most of the flow is nearly 1-D and can be realistically modeled by a set of ordinary differential equations (ODEs) that represent the conservation equations for a variable-area duct with friction and wall heat transfer. However, the region surrounding the fuel jet near each injector is best represented by the 3-D turbulent combustion of a jet in crossflow. We developed a reduced-order modeling strategy that combines the known 3-D scaling relations for a jet in crossflow with a set of flamelet lookup tables. An assumed PDF approach is used to include the appropriate 3-D turbulence properties in a statistical sense and the local interaction of turbulence with finite rate chemistry. We then spatially average the 3-D reaction rates over planes perpendicular to the main flow direction, which gives us a lookup table of the resulting $1-D$ reaction rates. These values are inserted into the source terms in the differential equations for species conservation in the solution of the ODEs describing the main flow. Using this approach, the main flow can be treated as a 1-D flow, but the local 3-D turbulent combustion and mixing around each fuel jet can be computed, and their influence on the overall heat release can be retained.

To develop a ROM formulation for computationally efficient application in MDO, several modeling assumptions are introduced. The main assumption arises from presenting the fuel injection and flame structure through the self-similar solution of a jet in crossflow. Currently, we only consider the perpendicular injection of fuel in a crossflow, and the self-similar modeling constants are determined from low-speed experiments. However, it is noted that this model can be improved as further results for scaling relations under high-speed flow conditions become available. Another potential model limitation arises from the flamelet assumption, in which a twostream combustion process between fuel and oxidizer is considered. Multistream systems that arise, for instance, by injecting different fuels or the successive mixing of different reactants currently are not considered. However, the flamelet model can be extended to account for more complex information by considered multistream and nonadiabatic processes $[17,18]$. In the present work, hydrogen/ oxygen combustion is considered. More complex mechanisms and hydrocarbon combustion can be incorporated in a straightforward way without increase in computational complexity of the ROM formulation.

\section{A. Conservation Equations}

For the description of a stationary turbulent reacting flow, a Reynolds-averaged approach is employed, in which an instantaneous flowfield quantity $\phi=\phi(t, \mathbf{x})$, for $\mathbf{x}=(x, y, z)$, is separated into mean and fluctuating contributions:

$$
\phi=\bar{\phi}+\phi^{\prime} \quad \text { with } \quad \bar{\phi}(\mathbf{x})=\frac{1}{T} \int_{t}^{t+T} \phi(t, \mathbf{x}) \mathrm{d} t
$$

and a corresponding Favre-averaged quantity is evaluated as $\tilde{\phi}=$ $\overline{\rho \phi} / \bar{\rho}$ and $\phi=\tilde{\phi}+\phi^{\prime \prime}$.

For the 1-D model, we assume that properties are homogeneous in each $y-z$ plane so that for each variable the value on that plane is equal to the area average of the variable. Note that in this model, $x$ is in the downstream axial direction in the combustor, $y$ is the upward transverse direction, and $z$ is the spanwise direction. The spatial average is defined by the following equation:

$$
\langle Q\rangle=\frac{1}{A} \iint_{A} Q \mathrm{~d} x \mathrm{~d} y
$$

where $A$ is the cross-sectional area. In general, however, we will refrain from writing the variables in angle brackets, $\langle\cdot\rangle$, to make equations easier to read. Unless otherwise specified, it should be assumed that variables are area-averaged.

MASIV solves for the 1-D evolutions of a set of ODEs for all flowfield quantities describing the combustion process. The derivation of these equations proceeds from well-known principles $[12,19]$, but some finer points deserve attention, so they are described in Appendix A. First, we consider the spatial derivative of the equation of state,

$$
\frac{1}{p} \frac{\mathrm{d} p}{\mathrm{~d} x}=\frac{1}{T} \frac{\mathrm{d} T}{\mathrm{~d} x}+\frac{1}{\rho} \frac{\mathrm{d} \rho}{\mathrm{d} x}-\frac{1}{W} \frac{\mathrm{d} W}{\mathrm{~d} x}
$$

to compute the pressure derivative, where $p$ is the pressure, $T$ is the temperature, $\rho$ is the density, and $W$ is the molecular weight of the mixture. Next, we employ the mass conservation equation,

$$
\frac{1}{\rho} \frac{\mathrm{d} \rho}{\mathrm{d} x}=\frac{1}{\dot{m}} \frac{\mathrm{d} \dot{m}}{\mathrm{~d} x}-\frac{1}{u} \frac{\mathrm{d} u}{\mathrm{~d} x}-\frac{1}{A} \frac{\mathrm{d} A}{\mathrm{~d} x}
$$

to find the density derivative, where $\dot{m}$ is the total mass flow rate in the duct, and $u$ is the velocity. Next, we use the species conservation equation,

$$
\frac{\mathrm{d} Y_{i}}{\mathrm{~d} x}=\frac{\dot{\omega}_{i}}{\rho u}+\frac{1}{\dot{m}} \frac{\mathrm{d} \dot{m}_{i}}{\mathrm{~d} x}-\frac{Y_{i}}{\dot{m}} \frac{\mathrm{d} \dot{m}}{\mathrm{~d} x}
$$

in order to account for mass addition to and reaction in the duct. In Eq. (5) $Y_{i}$ is the mass fraction of each species, $\dot{\omega}_{i}$ is the volumetric mass generation rate of each species, and $\mathrm{d} \dot{m}_{i} / \mathrm{d} x$ is the rate of mass addition through the walls for each species. Next, we use the momentum conservation equation,

$$
\frac{1}{u} \frac{\mathrm{d} u}{\mathrm{~d} x}=-\frac{1}{\rho u^{2}} \frac{\mathrm{d} p}{\mathrm{~d} x}-\frac{C_{f}}{2 A} \frac{\mathrm{d} S_{w}}{\mathrm{~d} x}-\frac{(1-\varepsilon)}{\dot{m}} \frac{\mathrm{d} \dot{m}}{\mathrm{~d} x}
$$

to find the derivative of axial velocity. A source term accounts for momentum carried into the duct by mass added through the walls. Here $C_{f}$ is the wall friction coefficient and $\mathrm{d} S_{w} / \mathrm{d} x$ is the rate of change of total wetted area with downstream distance. The direction parameter $\varepsilon$ is $u_{\mathrm{inj}, x} / u$, where $u_{\mathrm{inj}, x}$ is the $x$ component of the injected gas. Values of 1 and 0 correspond to parallel and perpendicular injection, respectively. The second term on the right-hand side represents friction forces and the third term represents momentum added through the walls. Finally, we use the energy conservation equation,

$$
\begin{gathered}
\frac{1}{h_{0}} \frac{\mathrm{d} T}{\mathrm{~d} x} \sum_{i} c_{p, i} Y_{i}=-\frac{u}{h_{0}} \frac{\mathrm{d} u}{\mathrm{~d} x}-\frac{1}{\dot{m}} \frac{\mathrm{d} \dot{m}}{\mathrm{~d} x}+\frac{1}{h_{0} \dot{m}} \frac{\rho u C_{f}\left(h_{\mathrm{aw}}-h_{w}\right)}{2 P r^{2 / 3}} \frac{\mathrm{d} S_{w}}{\mathrm{~d} x} \\
+\frac{1}{h_{0} \dot{m}} \frac{\rho u^{3} C_{f}}{2} \frac{\mathrm{d} S_{w}}{\mathrm{~d} x}-\frac{1}{h_{0}} \sum_{i} h_{i} \frac{\mathrm{d} Y_{i}}{\mathrm{~d} x}+\frac{1}{h_{0} \dot{m}} \sum_{i} h_{i} \frac{\mathrm{d} \dot{m}_{i}}{\mathrm{~d} x}
\end{gathered}
$$

to find the rate of change of temperature in the duct. In Eq. (7) $h_{0}$ is the stagnation enthalpy of the flow and $c_{p, i}$ is the specific heat at constant pressure of each species. Note that these equations consider all sources of enthalpy including sensible and chemical. As required by the Reynolds analogy, $h_{\text {aw }}$ is the enthalpy of the gas at an adiabatic wall and $h_{w}$ is the gas enthalpy at the wall temperature. Several source terms describe the addition of energy to the duct by friction, wall heat addition and chemical reaction. The left-hand side of the equation represents the energy-normalized rate of change of temperature. The third term on the right-hand side represents heat lost to the walls, which is computed using the Reynolds analogy. The fourth term represents work done by wall friction. The fifth term represents heat added by combustion and the sixth term represents energy added to the volume by mass addition through the walls.

Equations (3-7) represent $4+n$ equations for $4+n$ unknowns $\left(p, \rho, Y_{i}, u, T\right)$, where $n$ is the number of species considered. In the 
present implementation of the MASIV model, we have included nine species, corresponding to $\mathrm{H}_{2} /$ air chemistry with no nitrogen products, although any chemistry set can be used in principle.

\section{B. Assumed-Mixing Model}

We developed a mixing ROM in order to compute the chemical reaction rates $\left(\dot{\omega}_{i}\right)$ for each species, which are required in Eq. (5). The ROM simulates the 3-D mixing and 3-D turbulent combustion processes for a fuel jet that is injected perpendicular to an air crossflow. Then it reduces the 3-D local reaction rates to 1-D reaction-rate profiles by spatially averaging the computed 3-D reaction rates over each $y-z$ plane. This preserves the 3-D mixing/ combustion information and is an improvement over previous studies $[\underline{6}, \underline{7}, \underline{12}, \underline{19}, 20]$ that have unrealistically simulated the chemical reactions and mixing to be 1-D processes. At each spatial (x) location, the mean chemical reaction rate of each species is computed by combining the mixture fraction and fluctuation information with flamelet lookup tables.

In general, there are two ways to represent combustion: either as a reaction-rate-limited process (premixed), or as a mixing-limited process. Previous work $[6,7,12,19,20]$ has assumed that scramjet combustion is a reaction-limited process; this assumption is only realistic if fuel is mixed far upstream of the combustion region, which often does not occur in practical devices. In reality, the conditions in a scramjet are mixing-limited. That is, fuel and air are not homogeneously mixed, but instead there is a stoichiometric contour that surrounds any 3-D fuel jet, and the combustion actually occurs near this stoichiometric contour.

To account for this mixing-limited condition, we assume that the flow around the injectors is well-approximated by a 3-D jet in crossflow. Turbulent mixing properties are generated using gradient information from the assumed jet profile, which then allows us to compute the local reaction rate for each species. We spatially average the resulting reaction-rate field to get $\left\langle\dot{\omega}_{i}\right\rangle$ once the local reaction rates (including turbulent effects) have been modeled.

The first step is to compute the jet mixing profiles. Rather than store CFD information directly in lookup tables or POD basis functions, we use physical self-similarity arguments to rapidly create a solution. These scaling relations are algebraic relations that were determined experimentally by Hasselbrink and Mungal [21]. They represent the 3-D mean mixture-fraction field as function of $\mathbf{x}$ and give scaling relations for the other variables as functions of the mean mixture-fraction field. Note that although this scaling law is simple, it provides reasonable agreement for jet shape for a wide variety of conditions. The constants used in this study are from experiments on fully subsonic jets, but recent investigations [22] have examined transonic flows and found similar relations (although different values for some constants). Note that subsonic and supersonic mixing relations are similar, because the jet centerline and mixing-rate parameters are based on simple conservation arguments. However, we are ignoring certain dynamics such as barrel shocks in the injectant and shocks in the crossflow caused by injection, which some authors have identified [23].

The jet centerline penetration and spreading can be computed for each point using the jet scaling relationships [13]. This relationship is valid for momentum ratio $r_{u} \gg 1$, where $r_{u}$ is defined as

$$
r_{u}=\left[\frac{\rho_{\text {inj }}}{\rho}\left(\frac{u_{\text {inj }}}{u}\right)^{2}\right]^{1 / 2}
$$

and the injection density $\rho_{\text {inj }}$ and velocity $u_{\text {inj }}$ are known. The jet centerline path relationship

$$
\frac{y_{\mathrm{CL}}}{d_{\mathrm{inj}}}=c_{1}\left(\frac{x_{\mathrm{CL}}}{d_{\mathrm{inj}}}\right)^{c_{2}} r_{u}^{2 / 3}
$$

then traces the centerline path of the fuel jet. We assume that the fuel injector is choked, so the stagnation pressure and temperature of the fuel line are sufficient to compute conditions at the injector. The coordinates $x_{\mathrm{CL}}$ and $y_{\mathrm{CL}}$ define the centerline of the jet, and $d_{\mathrm{inj}}$ is the injector diameter.
The normalized concentration of injected fuel is given by another scaling relationship [21],

$$
\xi=c_{3}\left[\frac{\rho_{\text {inj }}}{\rho}\left(\frac{u_{\text {inj }}}{u}\right)^{-1}\left(\frac{x_{\mathrm{CL}}}{d_{\text {inj }}}\right)^{-2}\right]^{1 / 3}
$$

where $\xi$ is the ratio of the mole fractions of the streams. The mean mixture fraction $\tilde{f}_{\mathrm{CL}}$ is assumed to be 1 in the injected gas stream (pure fuel) and 0 in the crossflow (pure oxidizer):

$$
\begin{gathered}
r_{w}=\frac{W_{\text {inj }}}{W} \\
\tilde{f}_{\mathrm{CL}}=\frac{\xi r_{w}}{1+\left(r_{w}-1\right) \xi}
\end{gathered}
$$

where $r_{w}$ is the ratio of molecular weights of the injectant and crossflow.

The mixture fraction at a given point is determined by the centerline mixture fraction corresponding to that point, the jet spreading distance $(b)$, which is a function of distance from the injector along jet centerline $(s)$, and the distance from the jet centerline $(n)$ :

$$
\tilde{f}(s, n)=\tilde{f}_{\mathrm{CL}} \exp \left(\frac{-n^{2}}{2 b^{2}}\right)
$$

Values of $n$ and $b$ are computed using the following equations:

$$
\begin{gathered}
n^{2}=\left(x-x_{\mathrm{CL}}\right)^{2}+\left(y-y_{\mathrm{CL}}\right)^{2}+z^{2} \\
\frac{b}{d_{\mathrm{inj}}}=c_{4} r_{u}^{2 / 3}\left(\frac{x_{\mathrm{CL}}}{d_{\mathrm{inj}}}\right)^{c_{2}}
\end{gathered}
$$

Note that the orientation of the $(s, n)$ frame compared to the $(x, y, z)$ frame is shown in Fig. 1. Thus, by computing the shortest perpendicular distance from a given point to the jet centerline, the mixture fraction can be computed.

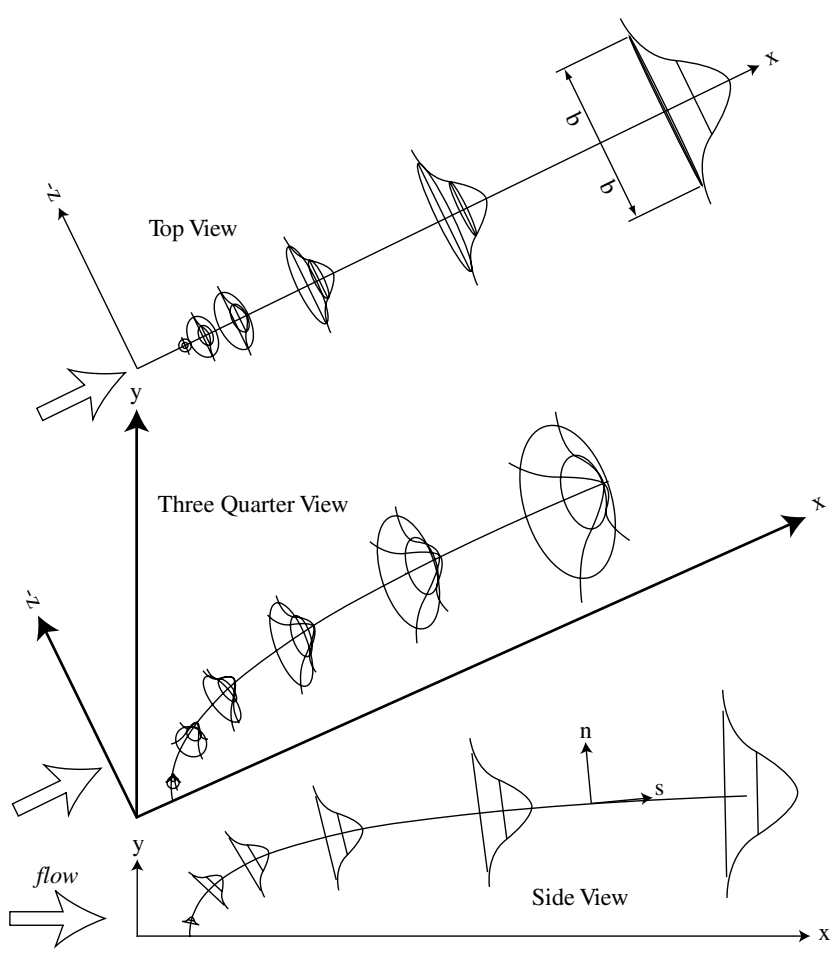

Fig. 1 Schematic of the spreading profile for a jet in crossflow. 
Table 1 Experimental constants for jet mixing model

\begin{tabular}{ccc}
\hline \hline Constant & Experimental range & MASIV value \\
\hline$c_{1}$ & 1.2 to $2.6[21]$ & 1.6 \\
$c_{2}$ & 0.28 to $0.34[\overline{21}]$ & $\left.\frac{1}{3}\right]$ \\
$c_{3}$ & $0.68-0.95[\underline{25}]$ & 1.3 \\
$c_{4}$ & $0.76[21]$ & 0.76 \\
$c_{5}$ & $0.0084-0.0093[\underline{25}]$ & 0.009 \\
\hline \hline
\end{tabular}

The mixture-fraction variance $\widetilde{f^{\prime \prime 2}}$ is computed using the Prandtl mixing-length argument. Measurements indicate that mixturefraction variance is essentially a function of the gradient of the mean mixture fraction, so that

$$
\sqrt{\widetilde{f^{\prime \prime 2}}}=\frac{c_{5}}{c_{4}} b|\nabla \tilde{f}|
$$

which is analogous to the mixing-length concept for velocity fluctuations [24]:

$$
\sqrt{\widetilde{u^{\prime \prime 2}}} \sim b|\nabla \tilde{u}|
$$

The constants $c_{1}-c_{5}$ are experimentally determined. Their values in MASIV are given in Table 1 . Note that we set $c_{3}$ in order to fit the experimental data as shown in Sec. IV. This is because the data of Hasselbrink and Mungal $[13,21]$ were measured in low-speed jets where diffusive effects are maximized, but the case in question is a high-speed ram case. In high-speed flows, especially transonic ones, some mixing is suppressed due to the speed and the shock pattern involved. Initial estimates based on more recent work on transonic (ram) cases [22] show values for $c_{3}$ that may be as high as 1.24.

Finally, we determine the mean scalar dissipation rate, $\tilde{\chi}$ using the formula

$$
\tilde{\chi}=2 D_{T}|\nabla \tilde{f}|^{2}
$$

where $D$ is the molecular diffusion coefficient and $D_{T}$ is the turbulent scalar diffusion coefficient. In the following, we model $D_{T}$ as [26]

$$
D_{T}=\frac{v_{T}}{S c_{T}}
$$

where $u_{\text {inj }} d_{\text {inj }} / v_{T}=45$. Here, the value of $u_{\text {inj }} d_{\text {inj }} / v_{T}$ is taken to be a tunable parameter, and the value 45 was found to give best agreement with the experimental results. Peters [26] suggests a value between 60 and 70 and Schlichting [27] suggests a value of 61; however, both of these are for low-speed jets, so the lower value we have chosen represents some mixing suppression due to the high speed of the flow in question. The turbulent Schmidt number is $S c_{T}=0.7$.

Figure 2 illustrates some profiles of mean mixture fraction, mixture-fraction variance, and reaction rate for the experimental conditions examined in this work. Note that the reaction rate depends on both the mean mixture fraction and the variance, indicating the roles of both fuel concentration and turbulent mixing on the reactionrate profile. Also, the mean mixture fraction and the mixture-fraction variance are low, because these planes are relatively far downstream from the injector.

\section{Flamelet Combustion Model for Diffusion Flames}

In the flamelet model, a turbulent diffusion flame is considered to be an ensemble of laminar flamelets $[14,26]$. At sufficiently large Damköhler number or sufficiently high activation energy, chemical

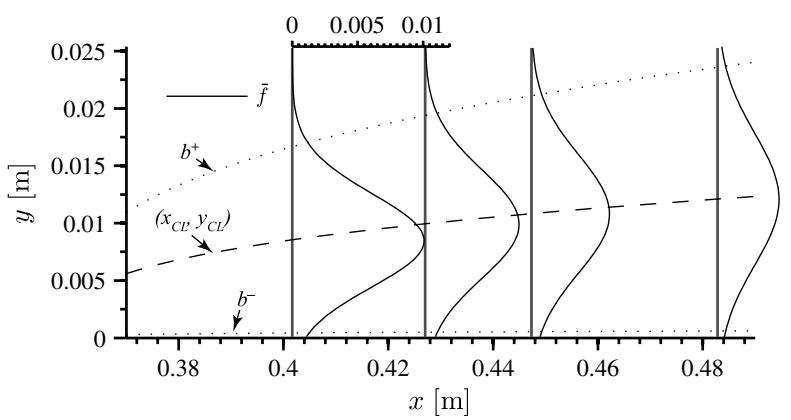

a) Mean mixture fraction, $\tilde{f}$ computed by MASIV

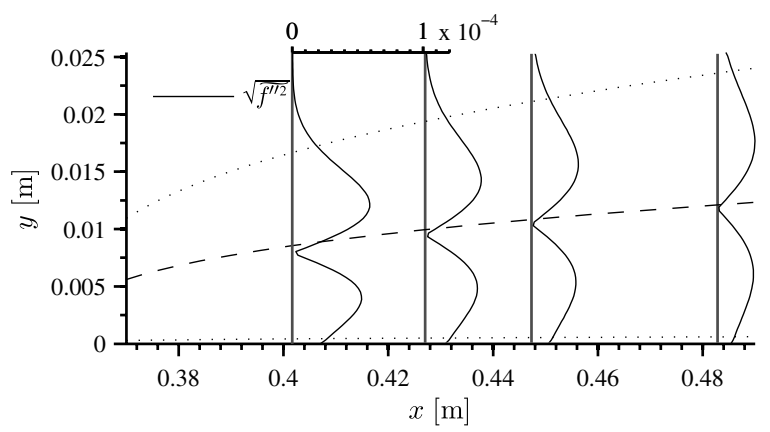

b) Mixture fraction RMS fluctuation, $\sqrt{\widetilde{f^{\prime \prime 2}}}$ computed by MASIV

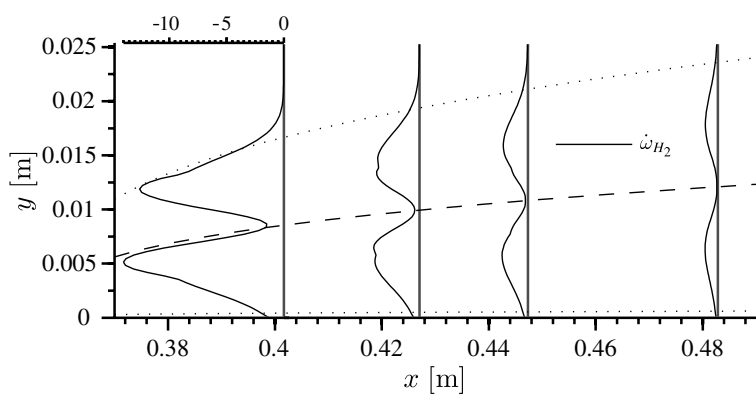

c) Production rate $\mathrm{H}_{2}, \dot{\omega}_{\mathrm{H}_{2}}$, in $\mathrm{kg} / \mathrm{m}^{3} / \mathrm{s}$

Fig. 2 MASIV computed profiles of mean mixture fraction, mixturefraction fluctuation and local rate of generation of $\mathrm{H}_{2}$ in the jet centerline $x-y$ plane. The $x$ locations shown are those marked in Fig. 4.

reactions and heat transfer occur in thin layers. If the characteristic length scale of these layers is smaller than that of the surrounding turbulence, the turbulent structures are unable to penetrate the reaction zone and are unable to destroy the flame structure. The effect of turbulence in this so-called flamelet regime results in a deformation and stretching of the flame sheet. With this notion, a

Table 3 Constant parameters used in the simulation

\begin{tabular}{cc}
\hline \hline Parameter & Value \\
\hline$P r$ & 0.71 \\
$T_{w}$ & $1100 \mathrm{~K}$ \\
$C_{f}$ & 0.003 \\
$r_{u}$ & 1.96 \\
$d_{\text {inj }}$ & $2.49 \mathrm{~mm}$ \\
\hline \hline
\end{tabular}

Table 2 Conditions at station 2a for experiment, FLUENT, and MASIV, with overall equivalence ratio $\phi=0.27$

\begin{tabular}{lcccccc}
\hline \hline & $T_{0}$ & $p_{0}$ & $T$ & $p$ & $u$ & Composition \\
\hline Vitiated-air crossflow & $1370 \mathrm{~K}$ & $333 \mathrm{kPa}$ & $1280 \mathrm{~K}$ & $261 \mathrm{kPa}$ & $458 \mathrm{~m} / \mathrm{s}$ & $Y_{\mathrm{O}_{2}}=0.251, Y_{\mathrm{N}_{2}}=0.611, Y_{\mathrm{H}_{2} \mathrm{O}}=0.138$ \\
Fuel jet & $298 \mathrm{~K}$ & $829 \mathrm{kPa}$ & $248 \mathrm{~K}$ & $438 \mathrm{kPa}$ & $1200 \mathrm{~m} / \mathrm{s}$ & $Y_{\mathrm{H}_{2}}=1$ \\
\hline \hline
\end{tabular}


flamelet can be considered as a thin reaction zone surrounded by a molecular transport layer, which, in turn, is embedded with a turbulent flow [28]. The structure of the flame in the flamelet regime can be described by the steady flamelet equations [14]:

$$
-\frac{\chi}{2} \frac{\partial^{2} \psi}{\partial f^{2}}=\dot{\omega}
$$

These equations can be derived from an asymptotic analysis of the conservation equations for species and energy, which are here denoted by the vector $\boldsymbol{\psi}=(\mathbf{Y}, h)^{T}$, and $\dot{\boldsymbol{\omega}}$ is the vector of the corresponding source terms. The scalar dissipation rate, appearing in Eq. (20), is modeled from the solution of a counterflow diffusion flame [29] and is expressed in terms of its value at stoichiometric mixture fraction and an analytical expression [29,30]:

$$
\chi=\chi_{\mathrm{st}} F(f)
$$

The solution of the steady flamelet equations can be represented by the so-called S-shaped curve [29], and all thermochemical quantities, collectively denoted by the vector $\phi=(\nu, \dot{\omega}, \ldots)^{T}$, can then be parameterized in terms of mixture fraction and scalar dissipation rate, viz.,

$$
\phi=\phi\left(f, \chi_{\mathrm{st}}\right)
$$

This state-space parameterization represents the instantaneous thermochemical quantities and does not account for turbulence/ chemistry interaction.

To account for the turbulence/chemistry interaction, the state relation (22) must be formulated for Favre-averaged quantities. These quantities are computed by employing a presumed joint PDF for mixture fraction and stoichiometric scalar dissipation rate:

$$
\tilde{\boldsymbol{\phi}}=\int_{0}^{\infty} \int_{0}^{1} \phi\left(f, \chi_{\mathrm{st}}\right) \tilde{P}(f) P\left(\chi_{\mathrm{st}}\right) \mathrm{d} f \mathrm{~d} \chi_{\mathrm{st}}
$$

where it is assumed that $f$ and $\chi_{\mathrm{st}}$ are statistically independent.

The marginal PDF of mixture fraction is modeled by a beta distribution [29], whose shape is fully characterized by the mean and variance of the mixture fraction. To model the distribution of the stoichiometric scalar dissipation rate, a log-normal distribution function is employed, which is presented as

$$
P\left(\chi_{\mathrm{st}}\right)=\frac{1}{\chi_{\mathrm{st}} \sigma \sqrt{2 \pi}} \exp \left\{-\frac{1}{2 \sigma^{2}}\left[\ln \left(\frac{\chi_{\mathrm{st}}}{\tilde{\chi}_{\mathrm{st}}}\right)+\frac{\sigma^{2}}{2}\right]^{2}\right\}
$$

in which $\sigma$ is the standard deviation of the PDF and is set to unity [31], and $\sigma$ is related to the stoichiometric scalar dissipation rate via

$$
\sigma^{2}=\ln \left(1+\frac{\widetilde{\chi_{\mathrm{st}}^{\prime \prime 2}}}{\widetilde{\chi_{\mathrm{st}}^{2}}}\right)
$$

With this, the Favre-averaged thermochemical state-space quantities can be expressed in terms of the first two moments of the mixture fraction and the mean stoichiometric scalar dissipation rate:

$$
\tilde{\boldsymbol{\phi}}=\tilde{\boldsymbol{\phi}}\left(\tilde{f}, \widetilde{f^{\prime \prime 2}}, \tilde{\chi}_{\mathrm{st}}\right)
$$

This gives $\dot{\boldsymbol{\omega}}\left(\tilde{f}, \widetilde{f^{\prime \prime 2}}, \tilde{\chi}_{\mathrm{st}}\right)$, which is mapped to $(x, y, z)$ space. Note that a minor inconsistency is introduced here, and the values obtained for species mass fractions in the 1-D integration will not necessarily correspond to the values obtained for local mass fractions in this step. To impose continuity and atom conservation on the flow, only the area-averaged reaction rates are used in the 1-D integration and not the local mass fractions as computed by the flamelet. Hence, the inconsistency is eliminated after the jet mixing and flamelet lookup step.

Next, we integrate $\dot{\omega}(x, y, z)$ to determine the one-dimensional rate of reaction of each species [recall Eq. (2)]:

$$
\left\langle\tilde{\dot{\omega}}_{i}\right\rangle(x)=\frac{1}{A} \iint \tilde{\dot{\omega}}_{i}(x, y, z) \mathrm{d} y \mathrm{~d} z
$$

These functions are tabulated for a given chemistry before the simulation.

\section{Solution Procedure}

We begin by considering the conservation equations and the equation of state [Eqs. (3-9)], solving them in the following manner. We avoid formulating the problem in terms of $c_{p}$ and directly use the enthalpy curve fits, which are available from NASA CEA $[32,33]$. We recommend the use of NASA CEA, because it uses many sources for its chemistry information and has current and extensive information.

Similarly, the sound speed $a$ for a reacting flow is defined as $a^{2}=(\partial p / \partial \rho)_{s}$, which produces different results for equilibrium and frozen flows [34]. This is because the variation of species mass fractions provides multiple paths for the state variables to follow. We avoid this difficulty by formulating the problem in terms of velocity rather than Mach number. This is what Heiser and Pratt [4] call enthalpy-kinetic energy $(H-K)$ space. It is more convenient than the typical temperature-Mach number $(T-M)$ space (which would require both $c_{p}$ and $a$ to be computed at solution time) and results in a simpler set of equations. We compute $a$ and $M$ in postprocessing after the solution has been found.

Other quantities indicated in Eqs. (3-7), which are required to solve the system, include the area profile $A(x)$ and its derivative $\mathrm{d} A / \mathrm{d} x$. The mass addition profile $\left(\mathrm{d} \dot{m}_{i} / \mathrm{d} x\right)(x)$ must also be given and can be summed to give

$$
\sum_{i} \frac{\mathrm{d} \dot{m}_{i}}{\mathrm{~d} x}=\frac{\mathrm{d} \dot{m}}{\mathrm{~d} x}(x)
$$

the total mass addition profile. For the integration step the reaction rates are pretabulated using the method described in Sec. II.C to yield $\dot{\omega}(x)$. We also assume a Prandtl number $P r$, a skin-friction coefficient $C_{f}$, and a wall temperature $T_{w}$.

Intermediate quantities are computed at each step:

$$
\dot{m}=\rho u A
$$

$$
\frac{\mathrm{d} W}{\mathrm{~d} x}=-W^{2} \sum_{i}\left(\frac{1}{W_{i}} \frac{\mathrm{d} Y_{i}}{\mathrm{~d} x}\right)
$$

$$
\begin{gathered}
\frac{\mathrm{d} S_{w}}{\mathrm{~d} x}=\sqrt{16 A+\left(\frac{\mathrm{d} A}{\mathrm{~d} x}\right)^{2}} \\
\frac{\mathrm{d} \dot{m}}{\mathrm{~d} x}=\sum_{i} \frac{\mathrm{d} \dot{m}_{i}}{\mathrm{~d} x}
\end{gathered}
$$

$$
h_{i}=h_{i}(T)
$$

$$
h=\sum_{i} Y_{i} h_{i}
$$

$$
h_{0}=h+\frac{u^{2}}{2}
$$

$$
h_{w}=h\left(T_{w}\right)
$$




$$
h_{\mathrm{aw}}=h+\sqrt{\operatorname{Pr}} \frac{u^{2}}{2}
$$

Once we have determined $\dot{\omega}(x)$, we have enough information to solve Eqs. (3-7). A stiff solver is required due to the rapid reaction rates. We used MATLAB's ode23tb because it is capable of handling stiff equations.

\section{Assessment of the Model}

To assess the accuracy of the MASIV model, we compared results to experimental data and to high-fidelity CFD solutions of the same configuration. We chose this approach because the three different results offer different insights and different levels of accuracy for certain parts of the flow. Not all quantities can be measured in the experiment, so although we treat it as a truth solution, not all variables of interest can be surveyed. The CFD model provides detailed information about all flow variables at all locations in the flow, but because some modeling of turbulence and chemistry is required, the simulation results can be skewed depending on modeling choices. Finally, the 1-D model can provide the most basic types of insights into conservation, jet mixing, and chemistry results in the duct. Differences between the methods are discussed below. We have attempted to match the simulations and the experimental results without tuning the models to the extent that their applicability to other problems is compromised. The nominal boundary conditions at the beginning of the duct for all three results are shown in Table 2. Note that these values are estimated from the experimental results.

\section{A. Experiment}

Previous supersonic combustion experiments were performed in a dual-mode ramjet/scramjet combustor [16]. This facility (shown in Fig. 3) supplies $21 \% \mathrm{O}_{2}$ mole-fraction vitiated air at stagnation temperatures $T_{0}$ up to $1500 \mathrm{~K}$. The test section is made of stainless steel and is shown in Fig. 1. A two-dimensional Mach 2.2 nozzle exits into a constant-area isolator with a cross section of 25.4 by $38.1 \mathrm{~mm}$. The constant-area isolator is followed by a wall-cavity flameholder and a nozzle section with a $4 \mathrm{deg}$ divergence angle. Room-temperature gaseous fuel was injected sonically through a single 2.49 -mm-diam port located on the test-section centerline $44.5 \mathrm{~mm}$ upstream of the cavity leading edge. Additional details on the facility and test section are available from Micka and Driscoll [35].

There are wall static pressure ports at 16 locations throughout the combustor and isolator. Estimates of the heat release distribution in the combustor were acquired using images of the $\mathrm{OH}^{*}$ luminosity [35]. Chemiluminescence is often used as a marker of the heat release in flames, and $\mathrm{OH}^{*}$ is proportional to the heat release rate in many cases [36]. The luminosity from $\mathrm{OH}^{*}$ was imaged using an Andor Istar intensified camera with a $310 \pm 10 \mathrm{~nm}$ bandpass interference filter.

\section{B. CFD Simulations}

The FLUENT boundary conditions are based on stagnation conditions in the air and fuel streams. Because of this, there is a discrepancy between the boundary conditions for FLUENT and MASIV, which corresponds to the difference between FLUENT's predictions of friction (and hence isolator performance) in the duct and the 1-D estimate of conditions from the experiment based on Heiser and Pratt's [4] isolator performance relationships.

The FLUENT CFDD geometry (shown in Fig. 4) is identical to that of the dual-mode combustor experiment described in the previous section. The meshed region includes the combustion and divergingarea sections of the experimental apparatus only, including only as much of the isolator section as required to contain the flow stagnation point upstream of the fuel injection port, and to allow for fuel diffusion upstream of the injector. The mesh is more densely clustered near the fuel injector to capture the physics of the fuel/air mixing as accurately as possible. The mesh also is denser near the leading and trailing edges of the stabilization cavity to capture their influence on the downstream thermal choke point created by heat addition to the subsonic flow. Symmetry about the vertical center plane allows us to reduce the computational requirement so that there are approximately 400,000 tetrahedral finite elements for half the combustor

We used the pressure-based solver in FLUENT, which solves the steady second-order upstream equations including viscosity and the energy equation; turbulence is modeled using the realizable $k-\epsilon$ model with standard wall functions. The mixture fraction and scalar dissipation rate are assumed to be statistically independent in the FLUENT implementation, so the joint $\operatorname{PDF} \tilde{P}\left(f, \chi_{\text {st }}\right)$ is set equal to $\tilde{P}(f) P\left(\chi_{\text {st }}\right)$. The PDF of the mixture fraction is assumed to be a beta function. Fluctuations in the scalar dissipation rate are ignored, so the

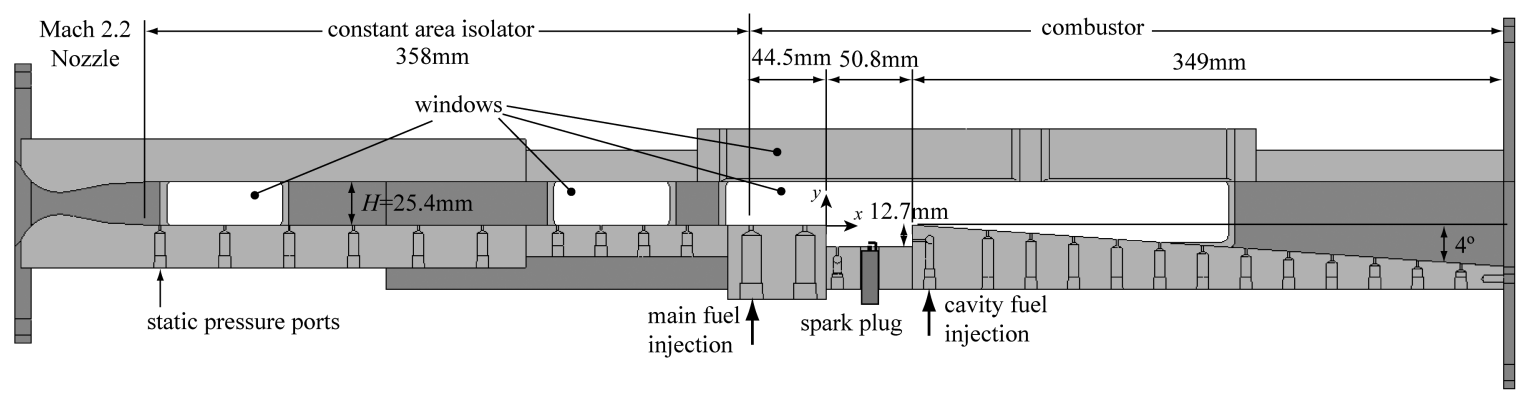

Fig. 3 Dual-mode combustor.

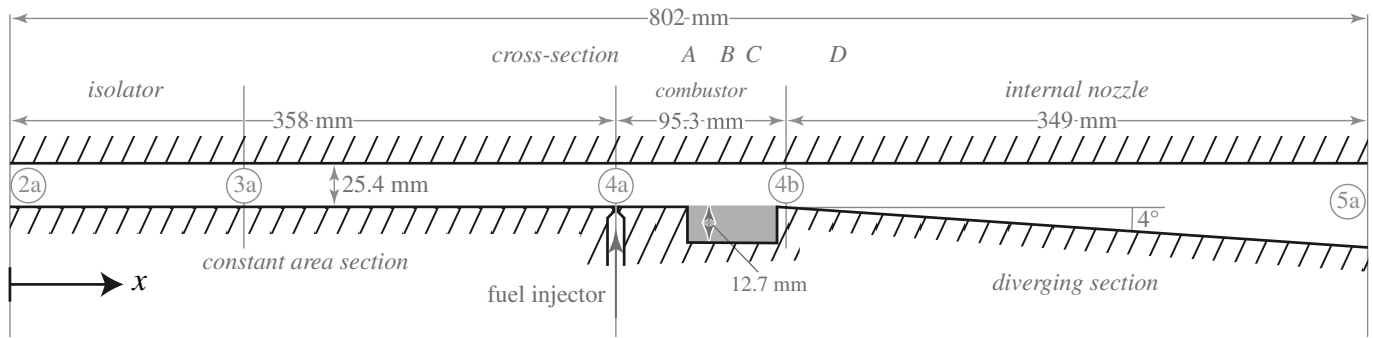

Fig. 4 Duct geometry for simulation. Note that only the experiment and FLUENT simulations include the cavity. MASIV simulations do not include cavity to avoid reversed-flow regions. 


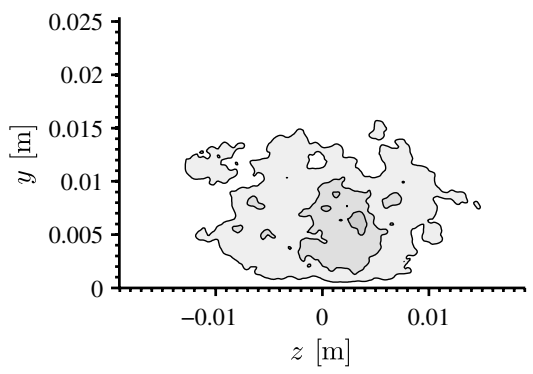

a) Cross section A, experiment

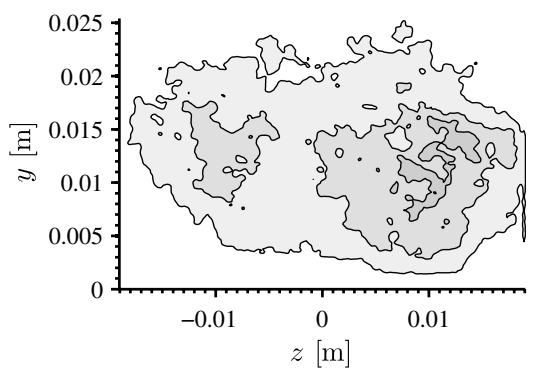

d) Cross section B, experiment

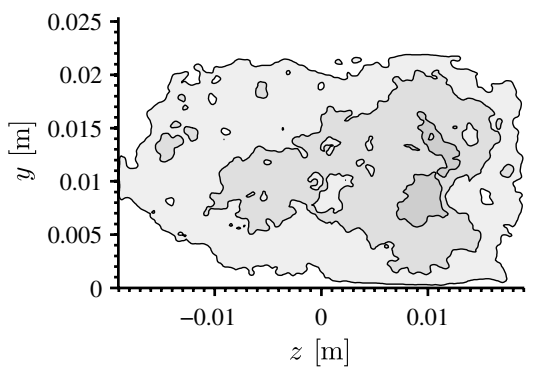

g) Cross section C, experiment

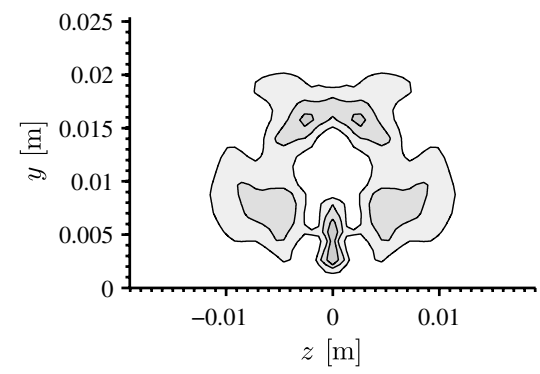

b) Cross section A, FLUENT

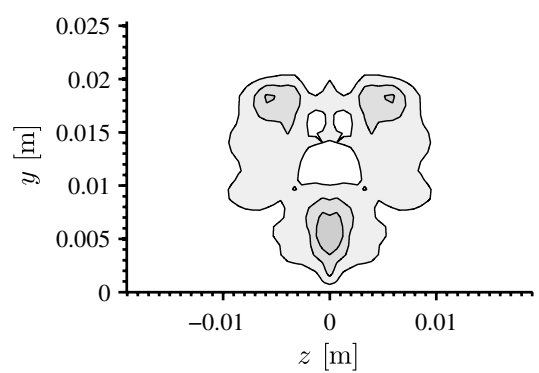

e) Cross section B, FLUENT

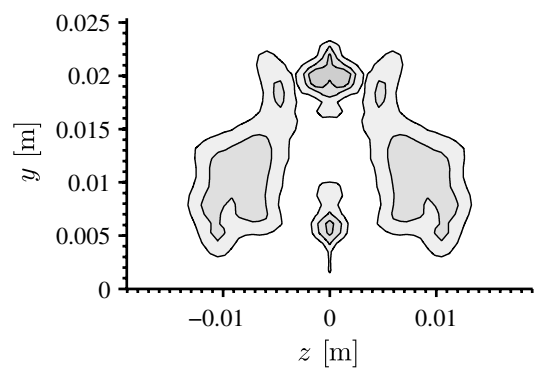

h) Cross section C, FLUENT

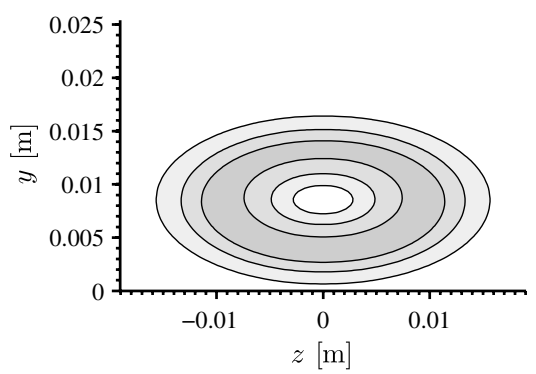

c) Cross section A, MASIV ROM

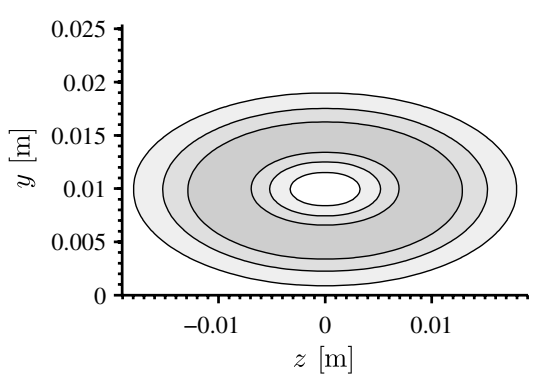

f) Cross section B, MASIV ROM

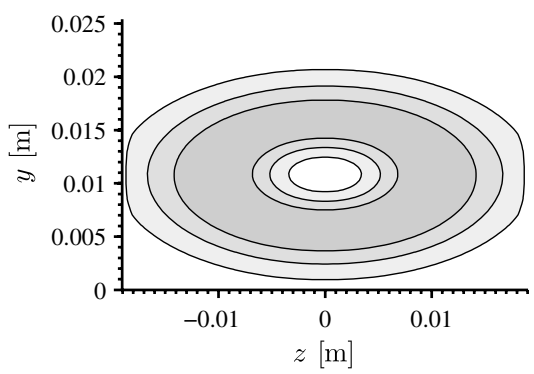

i) Cross section C, MASIV ROM

Fig. 5 Contours of heat release rate in $y-z$ cross sections at various $x$ locations. Each image is normalized by its own maximum value. Contours correspond to 25,50 , and $75 \%$ of the maximum value in each image. Locations of cross sections are marked in $\underline{4}$. The fuel injector is at $x=0.358 \mathrm{~m}$, and the $x$ locations are as follows. a-c) $x=0.402 \mathrm{~m}, \mathrm{~d}-\mathrm{f}$ ) $x=0.427 \mathrm{~m}$, and $\mathrm{g}-\mathrm{i}) \boldsymbol{x}=\mathbf{0 . 4 4 8} \mathrm{m}$.

PDF of $\chi$ becomes a delta function: $P=\delta\left(\chi_{\mathrm{st}}-\tilde{\chi}_{\mathrm{st}}\right)$. The mean scalar dissipation rate is modeled as $\tilde{\chi}_{\mathrm{st}}=2 \frac{\varepsilon}{k} \tilde{f}^{\prime 2}$, where $\bar{f}^{\prime 2}$ is the mean mixture-fraction variance [15]. Real-gas models are included to describe the compressible behavior of each of the species contained in the mixture. We used the nonpremixed, diabatic steady flamelet model including compressibility effects. A standard CHEMKIN [37] mechanism for $\mathrm{H}_{2}$ /air combustion dictates that the chemistry and $\mathrm{H}, \mathrm{O}, \mathrm{OH}, \mathrm{H}_{2} \mathrm{O}, \mathrm{H}_{2} \mathrm{O}_{2}$, and $\mathrm{HO}_{2}$ are the product species. The software-normalized residuals of the simulations were allowed to converge to values below $10^{-4}$ to ensure that the simulation has reached steady state.

\section{One-Dimensional Run Details}

The MASIV simulations use the same duct geometry (shown in Fig. 4) and initial conditions as were estimated from the experiment, except that the MASIV geometry uses a modified area profile that does not include the cavity, since a 1-D formulation cannot include regions of reversed flow. The values of the simulation constants in MASIV are shown in Table $\underline{3}$.

\section{Results}

Figures 5-12 provide a comparison of results from MASIV, FLUENT $\overline{\mathrm{C}} \mathrm{FD}$, and experimental data. We used three different metrics to tune the model. First, we looked at the jet spreading, which can be determined by examining cross-sectional $(y-z)$ planes, and compared the spreading characteristics of each of the three sources qualitatively. Second, looked at the flame length in the axial plane by comparing luminosity images from experiment to heat release images from the two numerical routines to ensure that the MASIV prediction is realistic. In these images we compared the shapes of the flame boundaries and total flame length. Third, we compared the axial 1-D luminosity from experiment to the 1-D heat release distributions predicted by the two codes. We expect the total heat release (area under the curve) to be similar among all three.

Contours of the heat release rate in various $y$ - $z$ planes are shown in Fig. 5. It is possible that differences between FLUENT and MASIV

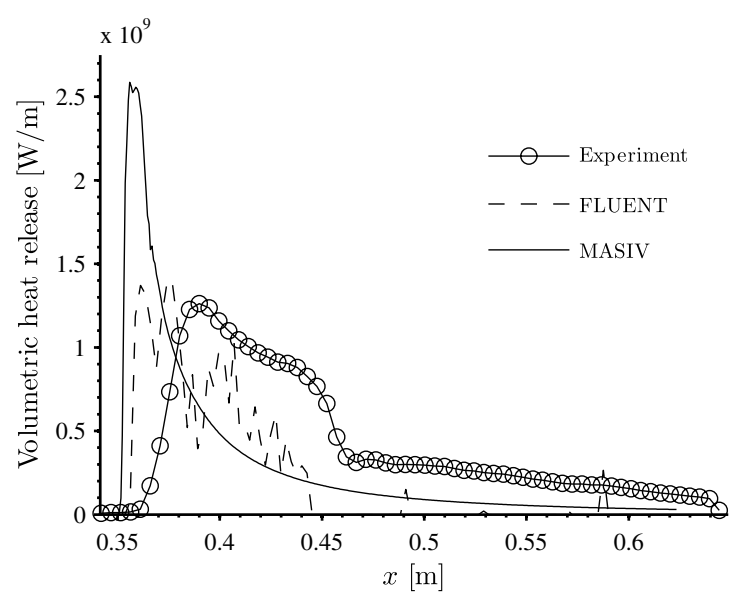

Fig. 6 Volumetric heat release rate for experiment, FLUENT, and MASIV. 


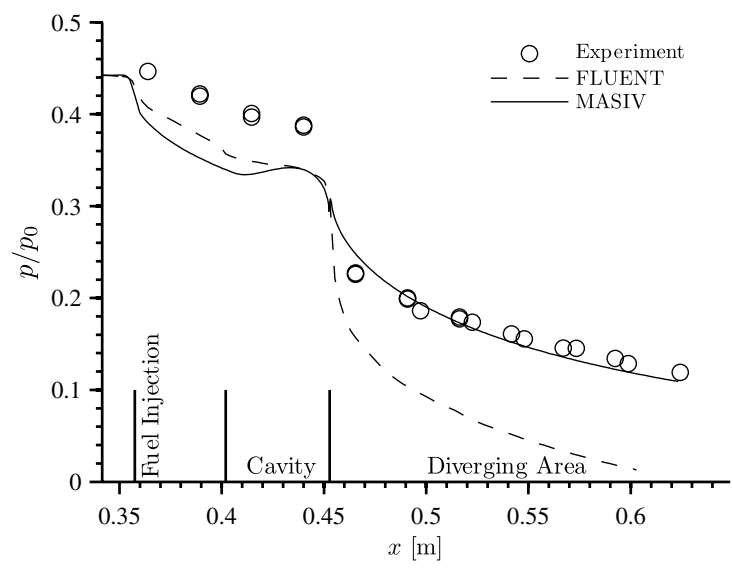

Fig. 7 Normalized pressure versus distance for experiment, FLUENT, and MASIV. Circles shown for the experiment represent measurements at individual pressure taps.

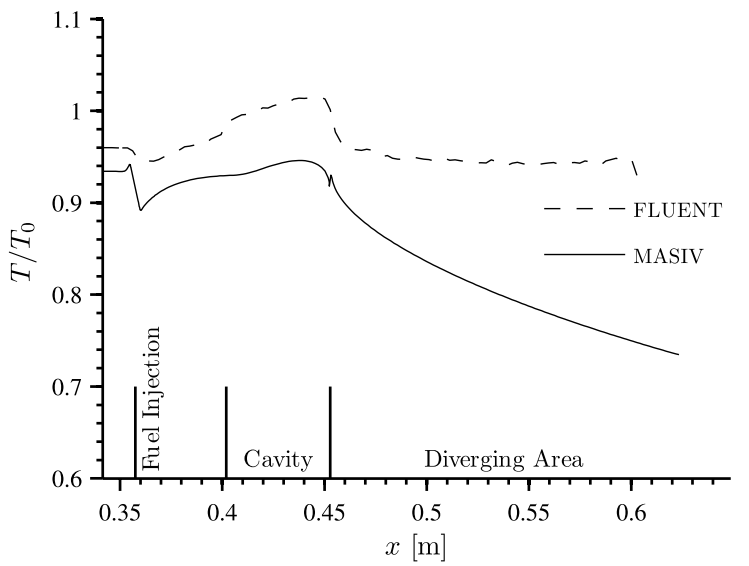

Fig. 8 Normalized temperature versus distance for FLUENT and MASIV.

are due to the simplified nature of the scaling relations ( $\underline{8}-16)$ and (18). However, there are some important considerations in evaluating the results. First, we note that the FLUENT results cannot necessarily be interpreted as a truth model, because they do not represent all aspects of the experimental flowfield accurately. We compare the results of FLUENT and MASIV, because they offer different insights into the problem, and we expect them to converge as modeling techniques used in each are improved.

Second, we note that each of the images is normalized to its own maximum, so they are only comparable to each other in a qualitative sense. The figure of merit for these images is that the loci of

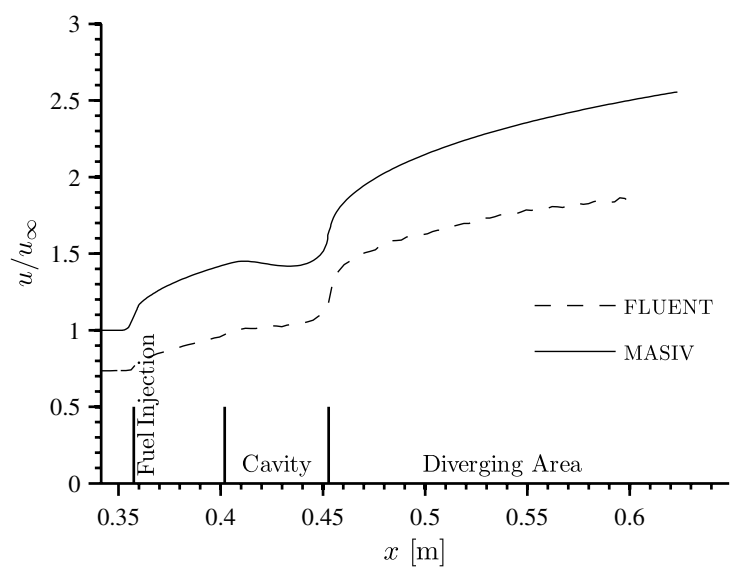

Fig. 9 Normalized velocity versus distance for FLUENT and MASIV.

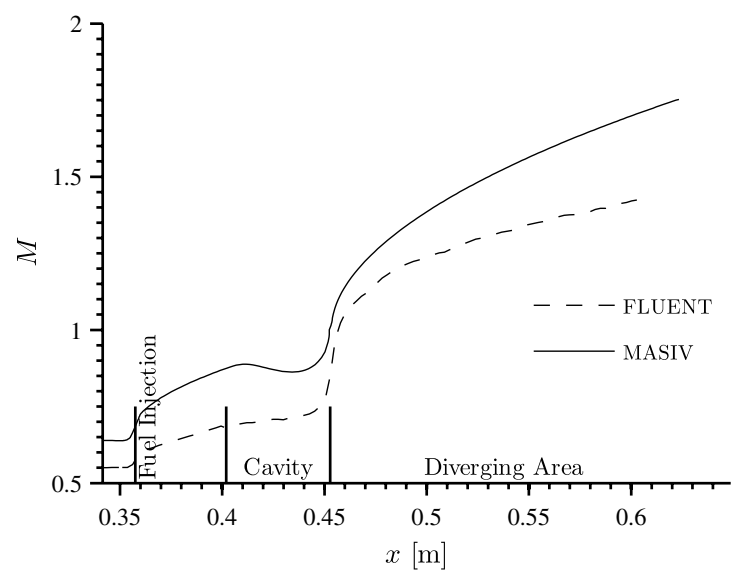

Fig. 10 Mach number versus distance for FLUENT and MASIV.

maximum reaction rate occur at approximately the same radial distances from the jet centerlines. This means that jet spreading computed by MASIV bears qualitative resemblance to the experiment and CFD cases, although the reaction rate it predicts is necessarily smeared annularly around the jet centerline due to the RANS formulation. Confinement has an effect on the centerline penetration and spreading of the jet, which is not included in the scaling relations of Hasselbrink and Mungal $[13,21]$. Experimental results suggest that the walls may prevent the jet from penetrating as far as it would otherwise.

The cross-sectional views are important because they give us a qualitative way to evaluate the model. We do not expect MASIV to reproduce the detailed structures seen in the experimental results, but we do expect the reaction contours to be approximately in the same area (and they appear to at least have the same trend) between the different models and we expect the regions of strongest reaction to be concentrated at the same distance away from the jet centerline. The FLUENT results at downstream locations are smaller in area than those of the experiment or MASIV, because FLUENT predicts some small regions of very high reaction rate toward the end of the duct.

Figure 6 shows that the MASIV model predicts stronger heat release very near the injector than does FLUENT. This is most likely because of the simplicity of the mixing model used in MASIV, which cannot truly predict the compressive effects of a high-speed crossflow impinging on a jet. This changes the mixing flowfield on the front side of the jet, and the resulting compression of the flame causes smaller heat release values.

Figure 7 shows that the pressure plots of numerical results differ from the experiment in the combustor region. They show that the computed pressure drops more rapidly than observed in reality. This is most likely due to heat being lost to the walls of the duct, or to momentum losses due to wall friction. The agreement between MASIV and experiment is probably somewhat coincidental, given

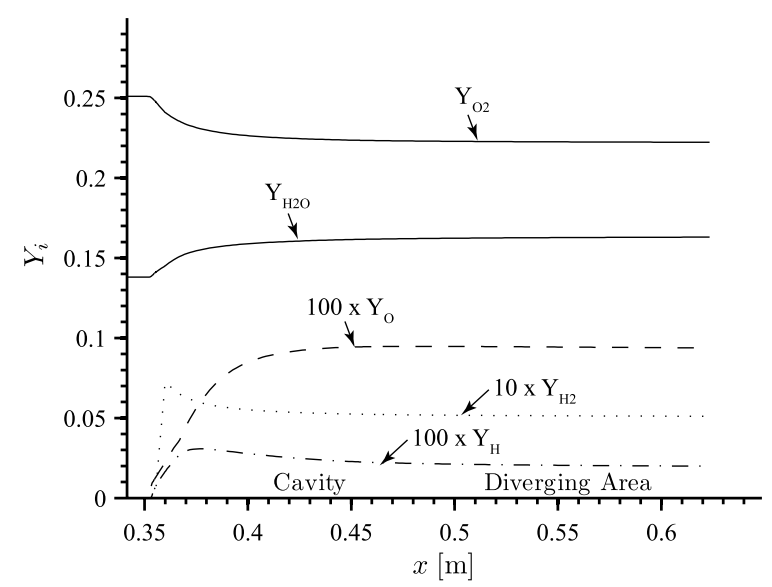

Fig. 11 Mass fraction versus distance for MASIV. 


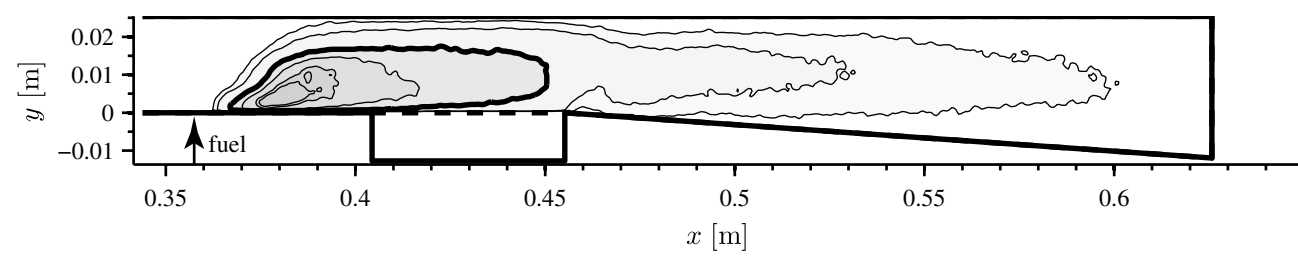

a) 2-D Side view-experiment. An $\mathrm{OH}$ fluorescence image that shows the estimated intensity of heat release

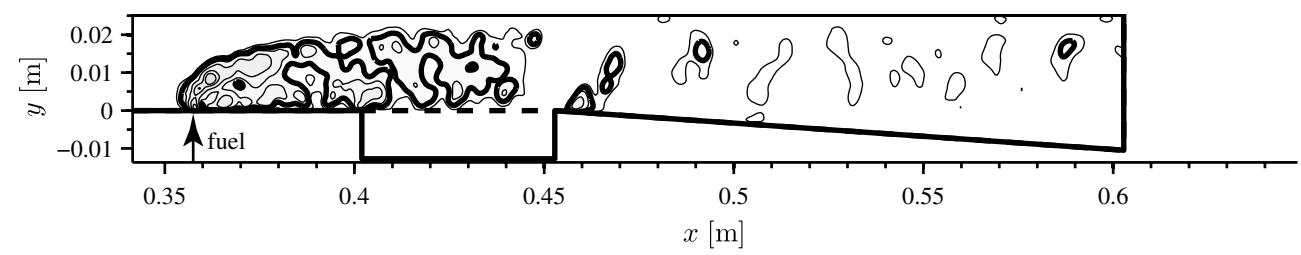

b) 2-D Side view-FLUENT. Heat release calculated as the positive part of the rate of change of sensible enthalpy not due to convection or diffusion

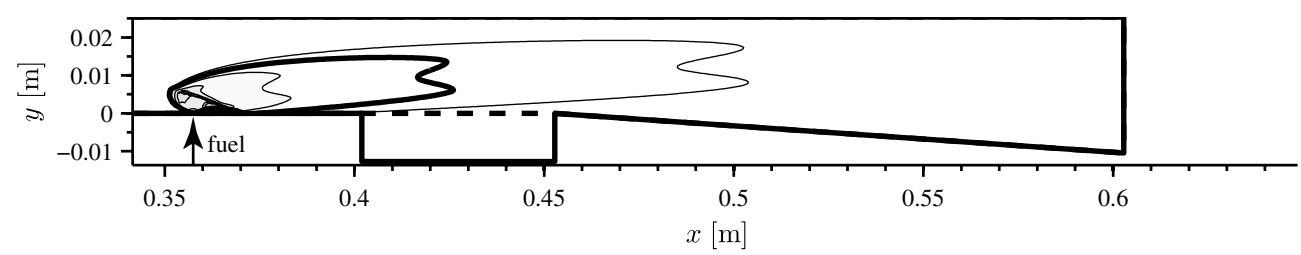

c) 2-D Side view-MASIV ROM. Heat release calculated as sensible enthalpy change due to reaction only

Fig. 12 Heat release results. The contours show isoclines containing 90, 75, 50, 25, 10, and $5 \%$ of the total heat release due to reaction in the duct. Comparison of corresponding isoclines of the three images shows predicted flame length from each. Bold contours show equivalent amounts of heat release, which are expected to have nearly the same length.

that both FLUENT and MASIV overpredict the pressure drop due to combustion from $x=0.35$ to $x=0.44$. One possible reason for the agreement of MASIV with experimental results in the aft section of the duct is that MASIV predicts that almost all of the reaction will occur inside the longest contour shown in Fig. 12c. The extra blobs of reaction predicted by FLUENT are accompanied by heat release, which will then drive down the pressure in that section. There may also be heat loss effects in this section of the combustor.

No experimental data were available for the temperature of the duct walls or wall friction, which has a strong effect on the result. Cold walls can remove a great deal of enthalpy from the flow, as can friction work. Experience suggests that the walls of the experiment will be slightly cooler than the vitiated-air stagnation temperature, since they will not have enough time to reach equilibrium with the flow. This means that the extra drop in pressure predicted by both codes is probably related to the fact that the heat loss to the walls is underpredicted.

Figure 8 shows the predicted temperature in the flow for FLUENT and MASIV. Again, heat loss to the walls can have a large effect on temperature, as well as differences in the predicted compositions of the flow. A small difference in predicted reaction rate can cause extra heat to be released due to additional burning of fuel or to recombination of radicals. Also, the more radicals in the flow (and the lower the molecular weight), the lower the temperature will be for a given enthalpy.

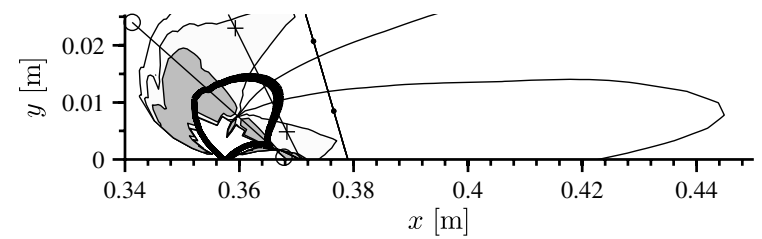

Fig. 13 Scalar dissipation-rate influence on stoichiometric contour $\left(f_{\text {st }}=\mathbf{0 . 0 3 1 6}\right)$. The contours of scalar dissipation rate represent $\chi=$ $\left[1 \mathrm{~s}^{-1}, 10 \mathrm{~s}^{-1}, 100 \mathrm{~s}^{-1}\right]$ from lightest to darkest. Note that $\chi=100 \mathrm{~s}^{-1}$ is the maximum scalar dissipation rate before quenching occurs. The mass fractions along lines marked with symbols $\bigcirc,+$, and $\bullet$ are shown in Fig. 14.
Figure 9 shows the velocity evolutions for FLUENT and MASIV. It is important to note that almost exactly the same trend is predicted by each model. However, the FLUENT code predicts a lower velocity than MASIV, because it predicts that heavier species make up a greater mass fraction than MASIV does. This, in turn, requires a greater velocity to satisfy continuity. Similarly, the Mach numbers in Fig. 10 follows the same trend, although the difference in predicted sound speed (again due to differences in predicted flow composition) brings the plots closer together than in Fig. 9 . The predicted thermal throat location is almost identical for the two models.

Figure 11 shows the MASIV-predicted mass fraction of several species of interest. It may be noted that the code predicts that much of the fuel will remain dissociated (which ties up combustion enthalpy) through the end of the duct. At these relatively low speeds this effect cannot be replicated with a finite-rate-limited (rather than mixinglimited, as here) solver.

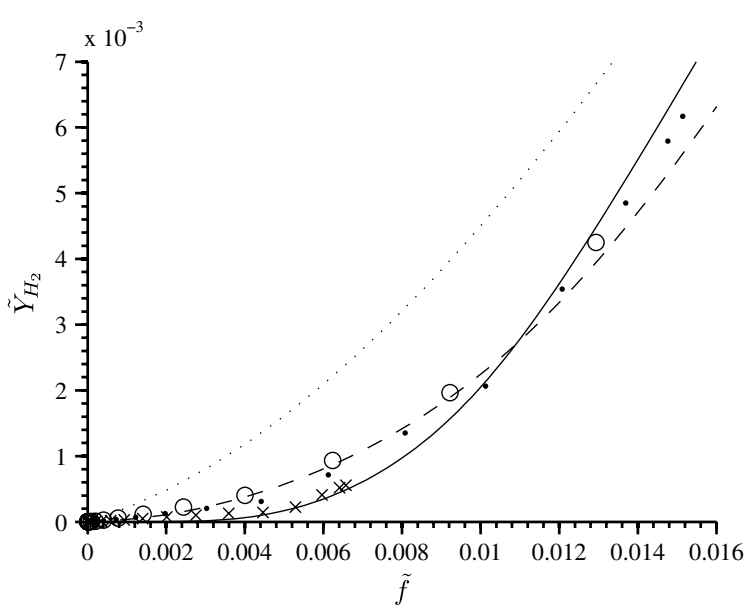

Fig. 14 Flamelets with $\chi=\left[1 \mathrm{~s}^{-1}, 10 \mathrm{~s}^{-1}, 100 \mathrm{~s}^{-1}\right]$. Symbols shown indicate the local mass fractions and mean mixture fractions around locations marked in Fig. 13. 
The heat release distributions shown in Fig. 12 show strong similarity between the flame shapes predicted by both codes. Since MASIV only includes turbulent effects in a statistical sense, none of the blobs of high combustion rate seen in the FLUENT result are present (Figs. 12b and 12c). However, the lengths of the various contours are similar in shape and length between the two models. Differences between simulation and experiment are most likely due to larger unsteady effects in the experiment, short experimental run time, diffusion and persistence of luminosity, and the high gains necessary to register the image.

Note that the MASIV model underpredicts the flame length, most likely due to the fuel being consumed so quickly near the injector. This is because the only way for the flame to be extinguished in the diffusion-flame model is for the strain rate to be excessively large and, as noted above, the strain on the front side of the jet is difficult to predict. In the experiment, the fuel not consumed on the front side of the jet convects downstream and burns there, contributing to the longer flame length. Figure 13 shows the influence of scalar dissipation rate on the flame. Since the fuel only has a high probability of burning at or very near to the stoichiometric contour, the strain rate at the stoichiometric contour is of primary importance. Note that the top front of the stoichiometric contour has very large strain rates, which correspond to incomplete combustion of the fuel.

The effect of scalar dissipation rate on the flamelet can be seen clearly in Fig. 14. This figure indicates that a large number of points in the flame react at conditions close to those of the $\chi=10 \mathrm{~s}^{-1}$ flamelet, which explains why not all the fuel is consumed.

\section{Conclusions}

The MASIV control-oriented propulsion code was found to agree with experimental heat release distribution measurements with accuracy comparable to that of the FLUENT CFD code. The MASIV code overpredicts the amount of reaction near injectors. This result is acceptable for control evaluation and MDO-type applications, because the overall behavior of the code is correct, even though there are some errors due to the simplified mixing model. Based on pressure values (the only variable that can be quantitatively compared for all 3 results) FLUENT gives a root-mean-square deviation of $25 \%$ from the experimentally measured pressures. MASIV gives an rms deviation of $18 \%$ from the experimentally measured pressures although it should be noted that the good agreement between MASIV and experiment in the diverging section is probably due to fortuitous choice of conditions and the balancing effect on pressure of simplified reaction contours due to the simplified mixing model and heat loss to walls. A typical MASIV run requires $\sim 1$ s of run time on a dual-core 32-bit computer.

While MASIV requires only a few seconds of run time on a modern personal computer to compute values of thrust and moments that agree with high-fidelity CFD results, it has several limitations. Currently, it is limited to 2-D steady (i.e., time-averaged) conditions. Fuel must be injected as a jet that is perpendicular to a crossflow of air. Our current implementation is limited to $\mathrm{H}_{2}$ fuel at one pressure and temperature for flamelet chemistry and ethylene $\left(\mathrm{C}_{2} \mathrm{H}_{4}\right)$ or methane $\left(\mathrm{CH}_{4}\right)$ chemistry for premixed finite rate chemistry. These limitations can easily be avoided in the future by creating additional flamelet lookup tables for different fuels and different reaction pressures and temperatures. To consider different injection angles, we would need experimentally verified scaling relations for jets injected at arbitrary angles into crossflows. Previously, we have computed the sensitivity of the thrust to the vehicle angle of attack, as well as the poles and zeros of the transfer functions that relate control inputs of fuel setting, elevator, and canard to 2-D airplane stability [7,9]. In the future, we will apply the MASIV code to optimization of proposed hypersonic vehicle configurations, in order to develop rules for creating stable and operable designs [10].

\section{Appendix A: Derivation of Conservation Equations}

The conservation equations for a 1-D flow in a variable-area duct have been derived many times in the literature $[\underline{12}, \underline{19}]$. We present here some points that require attention in order to calculate the effect of heat release and wall friction, as well as compute the Mach number of a reacting flow.

Figure A1 shows the canonical geometry of a duct control volume differential element. $\Phi$ in the figure represents a state variable of interest. The possible modes for addition of mass, momentum and energy are represented by arrows entering and leaving the control volume.

\section{Speed of Sound}

The two limits on sound speed are frozen and equilibrium. Beginning from Law's result [34] we obtain the following frozen sound-speed equation:

$$
a^{2}=R T \frac{\sum_{i} Y_{i} c_{p, i}}{\sum_{i} Y_{i} c_{p, i}-R}
$$

This is identical to the sound speed that can be found using the massweighted average $c_{p}$ and the mixture $R$. These in turn are found by the NASA CEA coefficients method as described above.

Here, the definition of $c_{p}$ corresponds to the frozen-flow case. We have found that using the frozen flow $c_{p}$ gives good results for prediction of the sonic point due to thermal choking, although it is unclear how much error is incurred by neglecting the effect of reaction at points other than the sonic point. The sonic point predicted using this method corresponds to within computer precision to the point at which the equations become extremely stiff, so it is useful for this purpose. Most likely, this is because any reaction-rate effects due to finite rate reactions will have derivatives that are small compared to the large derivatives of the state quantities near the sonic point. The specific heat $c_{p}$ of the mixture is not required for any other purpose, so the frozen-flow approximation is sufficient for the needs of the proposed algorithm.

\section{Molecular Weight}

$$
\begin{gathered}
\frac{1}{W}=\sum_{i} \frac{Y_{i}}{W_{i}} \\
\frac{\mathrm{d} W}{\mathrm{~d} x}=-W^{2} \sum_{i}\left(\frac{1}{W_{i}} \frac{\mathrm{d} Y_{i}}{\mathrm{~d} x}\right)
\end{gathered}
$$

\section{Evolution of Species}

We write a general form equation for the evolution of species allowing both creation of species in the volume due to reaction and addition of species through walls. This equation must be consistent with conservation of atoms, but Eq. (A5) does not guarantee this, so

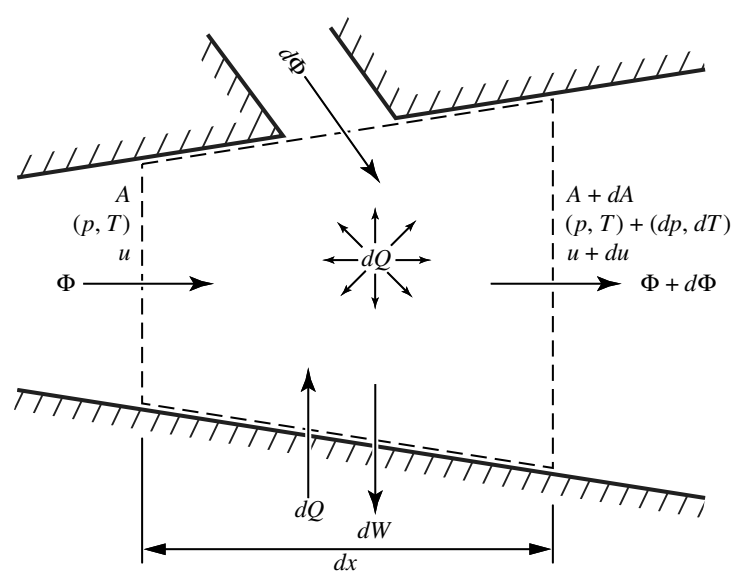

Fig. A1 Differential element for one-dimensional fluid flow; $\Phi$ is the flux of any quantity across the open system boundaries. 
the reaction rates $\dot{\omega}_{i}$ must themselves conserve atoms. Equation (A5) is consistent with conservation of mass and, indeed, the mass of each species is conserved when reactions that generate or consume species are considered:

$$
\begin{gathered}
\frac{\partial}{\partial t} \iiint_{V} Y_{i} \rho \mathrm{d} V+\iint_{S} Y_{i} \rho \mathbf{u} \cdot \mathbf{n} \mathrm{d} S=\int_{\delta x} \dot{\omega} A \mathrm{~d} x+\dot{m}_{i} \\
\frac{\mathrm{d} Y_{i}}{\mathrm{~d} x}=\frac{\dot{\omega}}{\rho u}+\frac{1}{\dot{m}} \frac{\mathrm{d} \dot{m}_{i}}{\mathrm{~d} x}-\frac{Y_{i}}{\dot{m}} \frac{\mathrm{d} \dot{m}}{\mathrm{~d} x}
\end{gathered}
$$

\section{Conservation of Momentum}

We begin with the control volume form of the momentum conservation equation, considering only steady solutions, neglecting body forces, and allowing momentum addition through the walls (assuming the added mass brings some momentum along with it). This results in the momentum equation (A7).

With the assumption that the wall friction is based on a constant coefficient of friction $C_{f}$, the wall shear stress can be represented as

$$
\tau_{w} \equiv C_{f} \rho u^{2} / 2
$$

The final momentum equation is then

$$
\frac{1}{u} \frac{\mathrm{d} u}{\mathrm{~d} x}=-\frac{1}{\rho u^{2}} \frac{\mathrm{d} p}{\mathrm{~d} x}-\frac{C_{f}}{2 A} \frac{\mathrm{d} S_{w}}{\mathrm{~d} x}-\frac{(1-\varepsilon)}{\dot{m}} \frac{\mathrm{d} \dot{m}}{\mathrm{~d} x}
$$

Here, $\varepsilon$ is the ratio of the axial velocity of the added mass to the axial velocity of the free stream.

One basic approximation for the wetted area $S_{w}$ is to consider square cross sections:

$$
\mathrm{d} S_{w}=\sqrt{16 A+\left(\frac{\mathrm{d} A}{\mathrm{~d} x}\right)^{2}}
$$

For anything more complex than that, it is best to simply record the wetted area at each point and to use it as a parameter, as with crosssectional area or mass addition.

\section{Conservation of Energy}

Total enthalpy of a gas mixture is the sum of the component enthalpies and the square of the velocity, assuming that all species have the same velocity:

$$
\begin{gathered}
h_{0}=\sum_{i}\left[h_{i}^{0}+\int_{T_{\mathrm{ref}}}^{T} c_{p, i}(\tau) \mathrm{d} \tau\right] Y_{i}+\frac{u^{2}}{2} \\
\frac{\mathrm{d} h_{0}}{\mathrm{~d} x}=u \frac{\mathrm{d} u}{\mathrm{~d} x}+\sum_{i}\left[c_{p, i} Y_{i} \frac{\mathrm{d} T}{\mathrm{~d} x}+h_{i} \frac{\mathrm{d} Y_{i}}{\mathrm{~d} x}\right] \\
\frac{1}{h_{0}} \frac{\mathrm{d} h_{0}}{\mathrm{~d} x}=-\frac{1}{\dot{m}} \frac{\mathrm{d} \dot{m}}{\mathrm{~d} x}+\frac{1}{h_{0} \dot{m}} \frac{\mathrm{d} \dot{Q}}{\mathrm{~d} x}+\frac{1}{h_{0} \dot{m}} \frac{\mathrm{d} \dot{\mathrm{d} x}}{h_{0}}+\frac{1}{h_{i}} \sum_{i} h_{i} \frac{\mathrm{d} \dot{m}_{i}}{\mathrm{~d} x} \\
\frac{1}{h_{0}}\left[u \frac{\mathrm{d} u}{\mathrm{~d} x}+\frac{\mathrm{d} T}{\mathrm{~d} x} \sum_{i} c_{p, i} Y_{i}+\sum_{i} h_{i} \frac{\mathrm{d} Y_{i}}{\mathrm{~d} x}\right]=-\frac{1}{\dot{m}} \frac{\mathrm{d} \dot{m}}{\mathrm{~d} x}+\frac{1}{h_{0} \dot{m}} \frac{\mathrm{d} \dot{\mathrm{d} x}}{\mathrm{~d} x} \\
+\frac{1}{h_{0} \dot{m}} \frac{\mathrm{d} \dot{W}}{\mathrm{~d} x}+\frac{1}{h_{0} \dot{m}} \sum_{i} h_{i} \frac{\mathrm{d} \dot{m}_{i}}{\mathrm{~d} x}
\end{gathered}
$$

We assume that heat addition per unit area is uniform and constant in the duct:

$$
\begin{gathered}
\dot{Q}=\dot{Q}^{\prime \prime} P_{w} \mathrm{~d} x \\
\dot{Q}=\dot{Q}^{\prime \prime} S_{w}
\end{gathered}
$$

$$
\mathrm{d} \dot{Q}=\dot{Q}^{\prime \prime} \mathrm{d} S_{w}
$$

If we suppose that the heat transfer is uniform and make use of the Reynolds analogy, assuming that $c_{p}$ of the gas mixture is uniform in each cross section,

$$
\begin{gathered}
C_{H}=\frac{\dot{Q}^{\prime \prime}}{\rho u\left(h_{\mathrm{aw}}-h_{w}\right)} \\
C_{H}=\frac{C_{f}}{2 P r^{2 / 3}} \\
\frac{\mathrm{d} \dot{Q}}{\mathrm{~d} x}=\frac{\rho u C_{f}\left(h_{\mathrm{aw}}-h_{w}\right)}{2 P r^{2 / 3}} \frac{\mathrm{d} S_{w}}{\mathrm{~d} x}
\end{gathered}
$$

The required enthalpies can be computed by the approximations

$$
\begin{gathered}
h_{\mathrm{aw}}=h+\operatorname{Pr} \frac{u^{2}}{2} \\
h_{w}=h\left(T_{w}\right)
\end{gathered}
$$

After some rearrangement to solve for the derivative of temperature, we obtain

$$
\begin{gathered}
\frac{1}{h_{0}} \frac{\mathrm{d} T}{\mathrm{~d} x} \sum_{i} c_{p, i} Y_{i}=-\frac{u}{h_{0}} \frac{\mathrm{d} u}{\mathrm{~d} x}-\frac{1}{\dot{m}} \frac{\mathrm{d} \dot{m}}{\mathrm{~d} x}+\frac{1}{h_{0} \dot{m}} \frac{\rho u C_{f}\left(h_{\mathrm{aw}}-h_{w}\right)}{2 \operatorname{Pr}^{2 / 3}} \frac{\mathrm{d} S_{w}}{\mathrm{~d} x} \\
+\frac{1}{h_{0} \dot{m}} \frac{\rho u^{3} C_{f}}{2} \frac{\mathrm{d} S_{w}}{\mathrm{~d} x}-\frac{1}{h_{0}} \sum_{i} h_{i} \frac{\mathrm{d} Y_{i}}{\mathrm{~d} x}+\frac{1}{h_{0} \dot{m}} \sum_{i} h_{i} \frac{\mathrm{d} \dot{m}_{i}}{\mathrm{~d} x} \quad(\mathrm{~A} 21)
\end{gathered}
$$

Note that the rate of change of wall area, $\mathrm{d} S_{w} / \mathrm{d} x$, can be computed using Eq. (A8) or some other method.

\section{Ratio of Specific Heats}

Although it does not appear in the above equations, sometimes the ratio of specific heats is desired. Since $R$ and $\hat{c}_{p}$ can be computed using quantities known at the time of solution via Eq. (A2), this results in a value of $\gamma$ that can be computed after the simulation is complete. Or, defining

$$
\hat{c}_{p}=\sum_{i} Y_{i} c_{p, i}
$$

we can make an approximation for $\gamma$.

$$
\gamma=\frac{c_{p}}{c_{p}-R}
$$

This is notable simply because it allows the ratio of specific heats to be computed as a function of the local state of the gas. A simpler approach will not give an accurate number because of the variation of $c_{p}$ with $x$.

\section{Acknowledgments}

This research was supported by U.S. Air Force Research Laboratory (AFRL) grant FA 8650-07-2-3744 for MACCCS (Michigan AFRL Collaborative Center for Control Science), monitored by David Doman and Michael Bolender. Support was also provided by NASA grant NNX08AB32A, administered by Donald Soloway, Technical Monitor.

\section{References}

[1] Bolender, M. A., and Doman, D. B., "Nonlinear Longitudinal Dynamical Model of an Air-Breathing Hypersonic Vehicle," Journal of Spacecraft and Rockets, Vol. 44, No. 2, 2007, pp. 374-387. doi: $10.2514 / 1.23370$ 
[2] Chavez, F. R., and Schmidt, D. K., "Analytical Aeropropulsive/ Aeroelastic Hypersonic-Vehicle Model with Dynamic Analysis," Journal of Guidance, Control, and Dynamics, Vol. 17, No. 6, 1994, pp. 1308-1319. doi: $10.2514 / 3.21349$

[3] Tarpley, C., and Lewis, M. J., "Stability Derivatives for a Hypersonic Caret-Wing Waverider," Journal of Aircraft, Vol. 32, No. 4, 1995, pp. 795-803. doi: $10.2514 / 3.46793$

[4] Heiser, W. H., and Pratt, D. T., Hypersonic Airbreathing Propulsion, AIAA, Washington, D.C., 1994.

[5] Dalle, D. J., Fotia, M. L., and Driscoll, J. F., "Reduced-Order Modeling of Two-Dimensional Supersonic Flows with Applications to Scramjet Inlets," Journal of Propulsion and Power, Vol. 26, No. 3, 2010, pp. $545-555$. doi:10.2514/1.46521

[6] Torrez, S. M., Scholten, N. A., Micka, D. J., Driscoll, J. F., Bolender, M. A., Doman, D. B., and Oppenheimer, M. W., "A Scramjet Engine Model Including Effects of Precombustion Shocks and Dissociation," 44th AIAA/ASME/SAE/ASEE Joint Propulsion Conference \& Exhibit, AIAA Paper 2008-4619, 2008.

[7] Torrez, S. M., Driscoll, J. F., Bolender, M. A., Oppenheimer, M. W., and Doman, D. B., "Effects of Improved Propulsion Modelling on the Flight Dynamics of Hypersonic Vehicles," AIAA Atmospheric Flight Mechanics Conference and Exhibit, AIAA Paper 2008-6386, 2008.

[8] Torrez, S. M., Driscoll, J. F., Dalle, D. J., and Micka, D. J., "Scramjet Engine Model MASIV: Role of Mixing, Chemistry and Wave Interaction," 45th AIAA/ASME/SAE/ASEE Joint Propulsion Conference and Exhibit, AIAA Paper 2009-4939, 2009.

[9] Torrez, S. M., Driscoll, J. F., Dalle, D. J., Bolender, M. A., and Doman, D. B., "Hypersonic Vehicle Thrust Sensitivity to Angle of Attack and Mach Number," AIAA Atmospheric Flight Mechanics Conference, AIAA Paper 2009-6152, 2009.

[10] Torrez, S. M., Driscoll, J. F., Dalle, D. J., and Fotia, M. L., "Preliminary Design Methodology for Hypersonic Engine Flowpaths," 16th AIAA/ DLR/DGLR International Space Planes and Hypersonic Systems and Technologies Conference, AIAA Paper 2009-7289, 2009.

[11] Hall, K. C., Thomas, J. P., and Dowell, E. H., "Proper Orthogonal Decomposition Technique for Transonic Unsteady Aerodynamic Flows," AIAA Journal, Vol. 38, No. 10, 2000, pp. 1853-1862. doi: $10.2514 / 2.867$

[12] O'Brien, T. F., Starkey, R. P., and Lewis, M. J., "Quasi-OneDimensional High-Speed Engine Model with Finite Rate Chemistry," Journal of Propulsion and Power, Vol. 17, No. 6, 2001, pp. 1366-1374. doi: $10.2514 / 2.5889$

[13] Hasselbrink, E. F., Jr., and Mungal, M. G., "Transverse Jets and Jet Flames. Part 1. Scaling Laws for Strong Transverse Jets," Journal of Fluid Mechanics, Vol. 443, 2001, pp. 1-25. doi:10.1017/S0022112001005146

[14] Peters, N., "Laminar Diffusion Flamelet Models in Non-Premixed Turbulent Combustion," Progress in Energy and Combustion Science, Vol. 10, 1984, pp. 319-339. doi:10.1016/0360-1285(84)90114-X

[15] FLUENT, Software Package, Ver. 6.3, Ansys, Inc., Canonsburg, PA, 2006.

[16] Micka, D. J., Torrez, S. M., and Driscoll, J. F., "Measurements and Modeling of the Heat Release Distribution in a Dual Mode Scramjet Combustor with Wall Fuel Injection," Proceedings of the 6th U.S. National Combustion Meeting [CD-ROM], 2009.

[17] Ihme, M., and See, Y. C., "LES Flamelet Modeling of a Three-Stream MILD Combustor: Analysis of Flame Sensitivity to Scalar Inflow Conditions," Proceedings of the Combustion Institute, Vol. 33, Combustion Inst., Pittsburgh, PA, 2010. doi:10.1016/j.proci.2010.05.019

[18] Ihme, M., and Pitsch, H., "Modeling of Radiation and Nitric Oxide Formation in Turbulent Nonpremixed Flames Using a Flamelet/ Progress Variable Formulation," Physics of Fluids, Vol. 20, 2008, Paper 055110. doi:10.1063/1.2911047

[19] Shapiro, A. H., Dynamics and Thermodynamics of Compressible Fluid Flow, Ronald Press, New York, 1953.

[20] Tetlow, M. R., and Doolan, C., "Comparison of Hydrogen and Hydrocarbon-Fueled Scramjet Engines for Orbital Insertion," Journal of Spacecraft and Rockets, Vol. 44, No. 2, 2007, pp. 365-372. doi:10.2514/1.24739

[21] Hasselbrink, E. F., Jr., and Mungal, M. G., "Transverse Jets and Jet Flames. Part 2. Velocity and OH Field Imaging," Journal of Fluid Mechanics, Vol. 443, 2001, pp. 27-68. doi: $10.1017 / \mathrm{S} 0022112001005158$

[22] Lin, K.-C., Ryan, M., Carter, C., Gruber, M., and Raffoul, C., "Raman Scattering Measurements of Gaseous Ethylene Jets in Mach 2 Supersonic Crossflow," Journal of Propulsion and Power, Vol. 26, No. 3, 2010, pp. 503-513. doi: $10.2514 / 1.43757$

[23] Billig, F. S., Corda, S., and Pandolfini, P. P., "Design Techniques for Dual Mode Ram-Scramjet Combustors," 7th National Aero-Space Plane Technology Symposium, Oct. 1989.

[24] Pope, S. B., Turbulent Flows, Cambridge Univ. Press, Cambridge, England, U.K., 2000.

[25] Smith, S. H., and Mungal, M. G., "Mixing, Structure and Scaling of the Jet in Crossflow," Journal of Fluid Mechanics, Vol. 357, 1998, pp. 83 122. doi:10.1017/S0022112097007891

[26] Peters, N., "Die Struktur Turbulenter Freistrahl-Diffusionsflammen," Chemie-Ingenieur-Technik, Vol. 55, No. 10, 1983, pp. 743-751. doi:10.1002/cite. 330551002

[27] Schlichting, H., and Gersten, K., Boundary Layer Theory, SpringerVerlag, New York, 2004.

[28] Williams, F. A., "Descriptions of Nonpremixed Turbulent Combustion," AIAA Paper 2006-1505, 2006.

[29] Peters, N., Turbulent Combustion, Cambridge Univ. Press, Cambridge, England, U.K., 2000.

[30] Ihme, M., and See, Y. C., "Prediction of Autoignition in a Lifted Methane/Air Flame Using an Unsteady Flamelet/Progress Variable Model," Combustion and Flame, Vol. 157, No. 10, 2010, pp. 1850 1862. doi:10.1016/j.combustflame.2010.07.015

[31] Effelsberg, E., and Peters, N., "Scalar Dissipation Rates in Turbulent Jets and Jet Diffusion Flames," Symposium (International) on Combustion, Vol. 22, Combustion Inst., Pittsburgh, PA, 1989, pp. 693 700 .

doi:10.1016/S0082-0784(89)80077-3

[32] McBride, B. J., and Gordon, S., "Computer Program for Calculation of Complex Chemical Equilibrium Compositions and Applications I. Analysis," NASA TR RP-1311, 1994.

[33] McBride, B. J., and Gordon, S., "Computer Program for Calculation of Complex Chemical Equilibrium Compositions and Applications II. User's Manual and Program Description," NASA TR RP-1311-P2, 1996.

[34] Law, C. K., Combustion Physics, Cambridge Univ. Press, Cambridge, England, U.K., 2006.

[35] Micka, D. J., and Driscoll, J. F., "Combustion Characteristics of a DualMode Scramjet Combustor with Cavity Flameholder," Proceedings of the Combustion Institute, Vol. 32, Combustion Inst., Pittsburgh, PA, 2009, pp. 2397-2404.

[36] Hardalupas, Y., and Orain, M., "Local Measurements of the TimeDependent Heat Release Rate and Equivalence Ratio Using Chemiluminescent Emission from a Flame," Combustion and Flame, Vol. 139, 2004, pp. 188-2007. doi:10.1016/j.combustflame.2004.08.003

[37] CHEMKIN, Software Package, Ver. 4.1, Reaction Design, San Diego, CA, 2006.

J. Powers

Associate Editor 